\title{
Patient self-report of oral health: Predicting the need for oral care
}

\author{
JB Epstein ${ }^{1 *}$, AL Watters ${ }^{2}$, D Villines ${ }^{3}$, C Conway 4 , C Jones ${ }^{5}$, G Schwartz ${ }^{5}$ and K Fung ${ }^{6}$ \\ ${ }^{1}$ Professor, Samuel Oschin Comprehensive Cancer Institute, Cedars-Sinai Medical Center, Los Angeles; Consultant, Division of Otolaryngology Head and Neck \\ Surgery, City of Hope National Medical Center, Duarte, CA, USA \\ ${ }^{2}$ Providence Cancer Center, Oral Oncology and Oral Medicine Clinic, Portland, Oregon, Clinical Assistant Professor, New York University College of Dentistry, \\ Department of Oral and Maxillofacial Pathology, Radiology and Medicine, New York, New York, USA \\ ${ }^{3}$ Director of Research, Advocate Health Care, Chicago, IL, USA \\ ${ }^{4}$ Private student, University of Toronto, Toronto, Canada, USA \\ ${ }^{5}$ Private practice, Ottawa, Canada \\ ${ }^{6}$ Private practice, Ottawa, Canada, Division of Dentistry/Oral \& Maxillofacial Surgery, Ottawa Hospital, Ottawa, Canada
}

\begin{abstract}
Background: People with complex medical conditions often experience barriers to oral care and are at an increased risk of developing dental disease, which may lead to local exacerbation, pain, and systemic infection. As access to primary medical care increases, improved screening methods by non-dental providers may address these barriers. This study investigates the predictive ability of a patient reported oral symptoms instrument to determine the need for urgent versus routine dental care.

Methods: Patients completed a questionnaire, querying, oral habits, dentate status, and symptoms of oral disease presence. An examiner determined the need for urgent versus routine care. Multivariate Logistic Regression was used to determine predictor variables in the model of urgent care needs and Area Under the Curve was used to determine discriminatory capabilities of the model.
\end{abstract}

Results: There were 114 urgent care and 890 routine cases $(\mathrm{n}=1004)$. The oral health composite score ranged from zero $(9.3 \%)$ to six $(0.1 \%)$ with the mean score of $1.62(\mathrm{sd}=0.98)$. The oral health composite score had modest diagnostic capability $(\mathrm{AUC}=0.759)$.

Conclusions: The patient reported oral symptoms is a novel, reliable instrument that may have utility for non-dental providers in various settings to identify urgent dental care needs based on patient reported symptoms.

\section{Introduction}

\section{Background}

Chronic dental diseases are among the most prevalent health problems worldwide and the most common childhood chronic illness in the United States [1]. Oral conditions such as periodontal disease and dental caries are most common and severe in populations that experience socioeconomic, social, cultural, and geographic barriers to care [2-7]. Novel strategies for intervention, including those aimed at increasing education and screening by primary care medical providers, have been suggested. People with limited access to oral health care may be at increased risk of having untreated dental disease which may place them at risk of local infection, pain, and systemic infection during medical therapy $[8,9]$.

In order to identify patients who require urgent dental and oral care needs, an accurate screening tool would be useful when oral conditions may be exacerbated during and following medical therapy and when effective dental management is not available. In the Institute of Medicine 2011 report on Improving Access to Oral Health Care for Vulnerable and Underserved Populations there was a call for a teambased, patient centered approach to care which can be met with various novel, inter-professional models [10]. However, physicians, nurses, and other non-dental providers lack the training necessary to diagnose dental disease or to make appropriate and timely referrals for treatment.
Recent public health efforts have focused on improved integration between medical and dental providers, as the number of practitioners in primary medical services vastly outweighs the number of oral health providers. However, medical providers are typically limited to palliative care for dental pain or infection $[11,12]$. The ability to identify patients with urgent dental disease before pain and infection is evident, without the use of dental radiographs or oral health trained providers, is essential. Patient reported outcome measures (PROMs) are frequently investigated in Quality of Life studies. As PROMs are integrated into clinical care, these instruments are increasingly used to detect disease burden in addition to assessing adequacy of symptom management. Many guidelines recommend an oral/dental evaluation prior to therapy for people with HNC, bone marrow/stem cell transplant, and chemotherapy by a dentist who has experience and expertise in treating and assessing patients undergoing cancer

Correspondence to: Joel B Epstein, , DMD, MSD, FRCD(C), FDS RCS(Ed), 8500 Wilshire Blvd, Suite 800, Beverly Hills, CA 90211, USA, Tel: 310 685-8500; E-mail: Joel.epstein@cshs.org

Key words: patient reported outcomes, oral/dental screening, identifying oral/ dental disease

Received: August 02, 2016; Accepted: August 15, 2016; Published: August 22, 2016 
treatment [13-17]. Unfortunately, dental providers with this specific knowledge are relatively few in number and many cancer centers, even National Cancer Institute designated comprehensive cancer centers, may not have access to these resources [13].

At present, an instrument that can reliably predict objective oral health needs from subjective patient reported measures is not available. Therefore, we initiated development of an instrument to capture patient reported oral health status and compared these results with objectively identified oral health status by trained dental providers. Specifically, the objective was to develop an instrument to distinguish patients who had urgent oral health needs from those patients without urgent oral health needs.

\section{Methods}

Consecutive new patients referred to a private group specialty periodontal practice in Ottawa, Canada were approached upon office registration, from July 2013 to January 2014. 1,257 patients were approached to take part in the study; 253 patients seen in the practice declined to participate, or failed to complete the questionnaire; therefore the final data set included 1,004 cases for analysis. Patients were asked to complete a brief questionnaire, the patient self-report of oral symptoms (PROS) tool, which captured seven categories including demographic information, oral habits, dentate status, oral pain, oral function, pathology, and disease presence (Appendix 1). The survey was developed with input of medical, radiation and surgical oncologists, and dentists and reviewed by clinically experienced oral oncology experts who provided modifications prior to use in this setting.

Information collected included age, gender, reason for visit, tobacco and alcohol use, dentate status, tooth brushing and flossing habits, denture use, and time of most recent dental visit. The respondents were asked questions regarding signs of dental disease such as problems with third molars, broken or loose teeth, broken fillings, swollen gingiva, and bleeding gingiva. Functional deficits were assessed though report of difficulty swallowing solid food, changes in speech or eating, para functional habits, limited jaw opening, and problems with dentures. Additionally, three pain questions including jaw or tooth pain when biting, oral pain in the past year, or current tooth or facial pain were asked along with potential symptoms of oral pathology including xerostomia, mass or swelling in the mouth or jaw, and sores in the mouth. Finally respondents were asked to rate their overall oral health status as good, fair, or poor.

Routine head and neck, oral, dental, and periodontal exams were then performed. Respondents were stratified into two groups by one of the five examiners who were blinded to the survey results: non-urgent care and urgent care needs based on the results of the oral examination. All participating examiners were periodontists who were not calibrated between each other. These examiners were all board certified specialists and used their best clinical judgment in order to dichotomize the study population. Patients who required intervention within one to two weeks to prevent pain and infection due to deep decay, severe loss of periodontal support, periapical pathology, and oral lesions were stratified into the urgent care group. The remaining patients were grouped as needing non-urgent/routine care.

\section{Statistical analysis}

Categorical and dichotomous variables are presented as frequencies with percentages and continuous variables are presented as means \pm standard deviation. Univariate logistic regression was used to determine independent predictors of the outcome variable (urgent care or non-urgent care) and were retained in the multivariate model. All variables were initially considered for the regression model as this was an exploratory analysis and a regression selection parameter was not used to assess a model with all parameters. Continuous variables that were statistically significant in the univariate screen analysis were transformed into dichotomous variables for clinical utility. Optimal cut-offs were determined with Receiver Operating Characteristic (ROC) curves, which plots the true positive rate against the false positive rate to display the trade-off between the sensitivity and specificity of the diagnostic test.

A model was developed based on results from the multivariate model with each symptom having a weight of one. Predictor variables that were statistically significant predictors received the same weight of one for having the risk factor in the equation and zero for not having the risk factor and the oral health composite score was determined. The discriminatory and diagnostic capability of the oral health composite score was assessed using ROC analysis. Results are reported as the Area under the Curve (AUC), which represents the probability that the oral health composite score result for a randomly chosen positive case, will exceed the result for a randomly chosen negative case. Analyses were performed using SPSS $22.0^{\circ}$ for Windows (IBM Corp., Armonk, NY).

\section{Results}

Demographic data, patient characteristics, and oral complication symptoms are shown by care group status in Table 1 . The urgent care group was older than the non-urgent care group $(52.27 \pm 15.24$ vs. $45.30 \pm 17.44, \mathrm{p}<0.01)$ and there were no between group differences for tobacco and alcohol use. Respondents in the urgent care group were more likely to rate their oral health as poor than in the non-urgent care group $(7.9 \%$ vs. $3.0 \%, \mathrm{p}=0.01)$. The urgent care group reported less regular tooth brushing $(4.4 \%$ vs. $1.1 \%, \mathrm{p}=0.01)$ along with problems with dentures $(6.1 \% v$ s. $2.0 \%, \mathrm{p}=0.01)$ and wisdom teeth $(13.2 \% v s$. $6.4 \%, \mathrm{p}<0.01)$ than in the non-urgent care group Additionally, pain was reported more in the urgent care group than in the non-urgent care group for all three pain variables: tooth pain within one year $(60.5 \%$ vs. $32.8 \%$, p < 0.01); facial pain $(34.2 \%$ vs. $10.8 \%, \mathrm{p}<0.01)$; jaw or tooth pain with biting $(28.1 \% v s .8 .5 \%, \mathrm{p}<0.01)$. Swelling in the mouth or jaw and swollen gums were more frequently reported in the urgent care group (than in the non-urgent care group $(27.2 \% v s .5 .6 \%, \mathrm{p}<0.01$ and $28.1 \%$ vs. $12.5 \%, \mathrm{p}<0.01$, respectively).

Additional oral symptoms also were more frequently reported in the urgent care group than non-urgent care group including: mouth sores $(19.3 \%$ vs. $6.0 \%, \mathrm{p}<0.01)$; change in speech or eating due to oral problems, $(17.5 \%$ vs. $7.3 \%, \mathrm{p}<0.01)$; loose teeth $(23.7 \%$ vs. $9.8 \%, \mathrm{p}<$ $0.01)$; bleeding in the mouth $(21.1 \%$ vs. $12.2 \%, \mathrm{p}<0.01)$, and broken teeth or filings $(33.3 \%$ vs. $16.6 \%, \mathrm{p}<0.01)$. Dentate versus non-dentate status, flossing, dentures, dentist visit within the past year, difficulty swallowing solid food, having a specific rather than comprehensive consultation, xerostomia, parafunctional habits, and limited jaw opening did not differ statistically between the groups.

\section{Multivariate predictors of urgent care needs}

Univariate logistic regression screening was performed for all variables in Table 1 and statistical significance was similar to the between group comparisons. Figure 1 displays selected odds ratio and corresponding $95 \% \mathrm{CI}$ for of each variable while controlling for the other variables in the multivariate model. Swelling in the mouth or jaw was the best predictor of needing urgent care $(\mathrm{OR}=4.09,95 \% \mathrm{CI}=2.15$ 
Table 1. Demographic Characteristic, Smoking and Alcohol Use, Oral Care Habits, And Symptoms Of Oral Complications for the Total Sample and by Urgent Care Groups.

\begin{tabular}{|c|c|c|c|}
\hline & $\begin{array}{c}\text { Total Sample } \\
\quad(n=1004)\end{array}$ & $\begin{array}{c}\text { Urgent Care } \\
(n=114)\end{array}$ & $\begin{array}{l}\text { Non-urgent Care } \\
\quad(\mathbf{n}=\mathbf{8 9 0})\end{array}$ \\
\hline Age & $46.08 \pm 17.33$ & $52.27 \pm 15.24$ & $45.30 \pm 17.44$ \\
\hline Gender (female) & $557(55.5 \%)$ & $60(52.6 \%)$ & $497(55.8 \%)$ \\
\hline Tobacco use & $109(10.9 \%)$ & $16(14.0 \%)$ & $93(10.4 \%)$ \\
\hline Pack/day & $1.02 \pm 1.33$ & $1.0 \pm 0.76$ & $1.03 \pm 1.43$ \\
\hline Alcohol use & $588(58.6 \%)$ & $62(54.4 \%)$ & $526(59.1 \%)$ \\
\hline Drink/day & $1.74 \pm 0.84$ & $2.08 \pm 0.82$ & $1.70 \pm 0.83$ \\
\hline \multicolumn{4}{|l|}{ Oral status } \\
\hline No teeth & $5(0.5 \%)$ & $1(0.9 \%)$ & $4(0.4 \%)$ \\
\hline Some teeth & $65(6.5 \%)$ & $11(9.6 \%)$ & $54(6.1 \%)$ \\
\hline Most teeth & $877(87.4 \%)$ & $94(82.5 \%)$ & $783(88.0 \%)$ \\
\hline \multicolumn{4}{|l|}{ Tooth brushing } \\
\hline Irregular & $15(1.5 \%)$ & $5(4.4 \%)$ & $10(1.1 \%)$ \\
\hline Once a day & $186(18.5 \%)$ & $25(21.9 \%)$ & $161(18.1 \%)$ \\
\hline Twice a day & $798(79.5 \%)$ & $82(71.9 \%)$ & $716(80.4 \%)$ \\
\hline \multicolumn{4}{|l|}{ Flossing } \\
\hline Never & $62(6.2 \%)$ & $11(9.6 \%)$ & $51(5.7 \%)$ \\
\hline Irregular & $417(41.4 \%)$ & $44(38.6 \%)$ & $372(41.8 \%)$ \\
\hline At least once a day & $502(50.0 \%)$ & $55(48.2 \%)$ & $447(50.2 \%)$ \\
\hline Dentures & $68(6.8 \%)$ & $11(9.6 \%)$ & $57(6.4 \%)$ \\
\hline Denture problem & $25(2.5 \%)$ & $7(6.1 \%)$ & $18(2.0 \%)$ \\
\hline Wisdom teeth problem & $72(7.2 \%)$ & $15(13.2 \%)$ & $57(6.4 \%)$ \\
\hline Seen Dentist w/in one year & $975(97.1 \%)$ & $112(98.2 \%)$ & $863(97.0 \%)$ \\
\hline Tooth pain w/in one year & $363(36.0 \%)$ & $69(60.5 \%)$ & $292(32.8 \%)$ \\
\hline Facial pain & $135(13.4 \%)$ & $39(34.2 \%)$ & $96(10.8 \%)$ \\
\hline Swelling in mouth or jaw & $81(8.1 \%)$ & $31(27.2 \%)$ & $50(5.6 \%)$ \\
\hline Mouth sores & $75(7.5 \%)$ & $22(19.3 \%)$ & $53(6.0 \%)$ \\
\hline Change in speech/ eating due to oral problems & $85(8.5 \%)$ & $20(17.5 \%)$ & $65(7.3 \%)$ \\
\hline Difficulty swallowing solid food & $22(2.2 \%)$ & $4(3.5 \%)$ & $18(2.0 \%)$ \\
\hline Loose teeth & $114(11.4 \%)$ & $27(23.7 \%)$ & $87(9.8 \%)$ \\
\hline Bleeding in mouth & $133(13.2 \%)$ & $24(21.1 \%)$ & $109(12.2 \%)$ \\
\hline Swollen gums & $143(14.2 \%)$ & $32(28.1 \%)$ & $111(12.5 \%)$ \\
\hline Broken teeth or filings & $186(18.5 \%)$ & $38(33.3 \%)$ & $148(16.6 \%)$ \\
\hline Consultation (specific) & $850(84.7 \%)$ & $100(87.7 \%)$ & $750(84.3 \%)$ \\
\hline Xerostomia & $109(10.9 \%)$ & $15(13.2 \%)$ & $94(10.6 \%)$ \\
\hline Grind teeth & $332(33.1 \%)$ & $36(31.6 \%)$ & $296(33.3 \%)$ \\
\hline Jaw or tooth pain with biting & $108(10.8 \%)$ & $32(28.1 \%)$ & $76(8.5 \%)$ \\
\hline Jaw opening limited & $60(6.0 \%)$ & $9(7.9 \%)$ & $51(5.7 \%)$ \\
\hline \multicolumn{4}{|l|}{ Self-report of oral health } \\
\hline Poor & $36(3.6 \%)$ & $9(7.9 \%)$ & $27(3.0 \%)$ \\
\hline Fair & $318(31.7 \%)$ & $40(35.1 \%)$ & $278(31.2 \%)$ \\
\hline Good & $644(64.1 \%)$ & $63(55.3 \%)$ & $581(65.3 \%)$ \\
\hline
\end{tabular}

Demographic characteristic, smoking and alcohol use, oral care habits, and symptoms of oral complications are presented as means \pm standard deviations for continuous variables and as frequencies with percentages for categorical variables. Data is presented for all subjects (total sample) and for the urgent and non-urgent care groups.

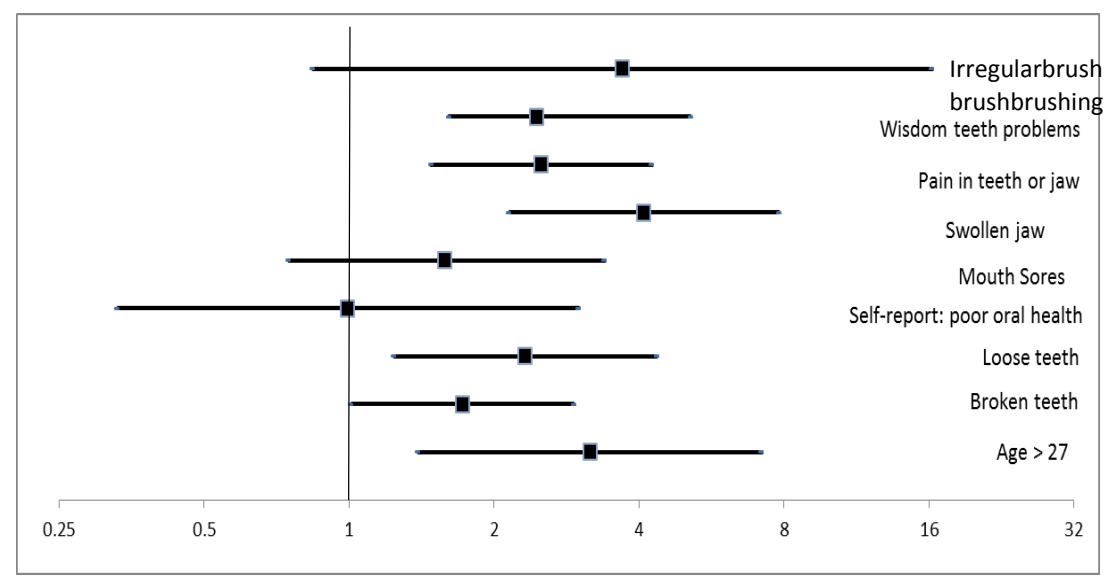

Selected odds ratio and corresponding $95 \%$ CI for of each variable while controlling for the other variables in the multivariate model. Variables that were statistically significant predictors in the univariate model were included in the multivariate model.

Figure 1. Multivariate odds ratios for predicting urgent care needs. 
- 7.81, $\mathrm{p}<0.01)$. Other variables that remained statistically significant predictors in the multivariate logistic regression model were wisdom teeth problems $(\mathrm{OR}=2.45,95 \% \mathrm{CI}=1.61-5.17, \mathrm{p}<0.01)$, jaw or tooth pain with biting $(\mathrm{OR}=2.50,95 \% \mathrm{CI}=1.48-4.25, \mathrm{p}<0.01)$, loose teeth $(\mathrm{OR}=2.32,95 \% \mathrm{CI}=1.24-4.35, \mathrm{p}<0.01)$, broken teeth or filings $(\mathrm{OR}$ $=1.72,95 \% \mathrm{CI}=1.01-2.93, \mathrm{p}<0.01)$ and age over 27 years old $(\mathrm{OR}=$ $3.16,95 \% \mathrm{CI}=1.39-7.19, \mathrm{p}<0.01)$.

\section{Diagnostic capability}

The oral health composite score was computed and included the following variables: swelling in the mouth or jaw, wisdom teeth problems, jaw or tooth pain with biting, loose teeth, broken teeth or filings and age $>27$ years old. Scores ranged from zero $(9.3 \%)$ to six $(0.1 \%)$ with the mean score of $1.62 \pm 0.98$. The oral health composite score had modest urgent care diagnostic capability $(\mathrm{AUC}=0.759)$ (Figure 2). The optimal cutoff was 1.5 corresponding to a sensitivity of 0.84 and a specificity of 0.55 .

\section{Discussion}

The study evaluated the potential utility of PRO to predict the urgency of dental treatment in an out patient population. The goal is to develop a PRO that may provide data suggesting the need for dental evaluation and treatment for use in medically complex populations. Our findings suggest utility in this outpatient population. Further development requires evaluation of this PRO in specific medically complex populations.

Responses of patients requiring urgent dental care were less likely to report their oral health as good than patients in the non-urgent dental care group. Predictors of urgent oral care needs, when controlling for other predictors, included swelling in the mouth or jaw, third molar problems, jaw or tooth pain with biting, loose teeth, broken teeth or filings and age $>27$ years old. Overall the strongest predictors of urgent oral care needs included swelling in the mouth or jaw, followed by age $>27$ years, third molar problems, jaw or tooth pain with biting and loose teeth (Figure 2).

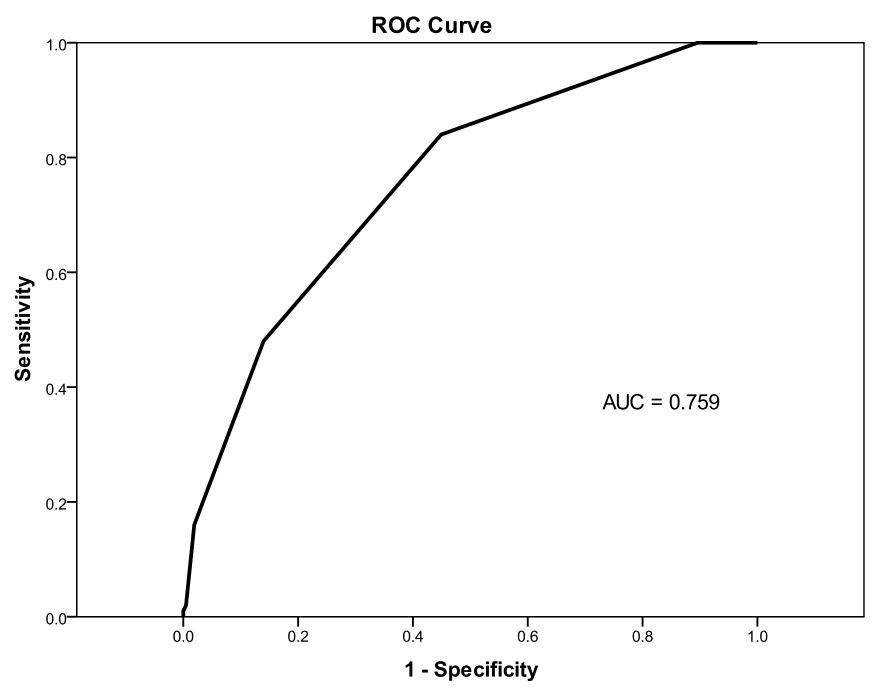

Oral Health Composite Score was computed as follows: predictor variables that were statistically significant predictors in the multivariate model received a weight of one for having the risk factor in the equation and zero for not having the risk factor and the oral health composite score was determined by summing the score.

Figure 2. Receiver Operating Characteristic Curves for Oral Health Composite Score with all Statistically Significant Predictors in the Oral Health Composite Score.
Creating a score from predictors allowed for modest diagnostic capability $(\mathrm{AUC}=0.704)$. As this instrument provides an adjunctive instrument for non-dental trained health care providers, moderate diagnostic capability may be acceptable in this clinical setting. It is not however, a substitution for a clinical dental examination. Future studies should address how the PROS instrument could be revised to provide higher diagnostic capability. The instrument should be assessed in the medical setting among patients with underlying medical comorbidities, those in whom dental conditions may require treatment prior to medical management, and in patients in which the medical therapy limits future dental treatment. Additionally, the instrument should be used to identify patients who require preventive protocols for maintenance of oral health following acute medical care.

The PROS tool was primarily developed for patients with solid tumors such as breast, colon, prostate and lung for use prior to cancer treatment, as there are no current guidelines that require oral and dental examinations before treatment. This is in contrast to patients with HNC and stem cell transplants, in which current guidelines recommend that all patients receive comprehensive dental, oral, and head and neck examinations and necessary treatment, prior to cancer treatment. For many other patients who are at high risk of complications from untreated dental disease, the PROS tool may play an important role in identifying urgent oral care needs in patients with medical conditions such as poorly controlled diabetes, patients with renal, cardiac and hepatic diseases, people admitted to long-term care facilities, and in patients planning to or currently receiving osteolytic inhibitors.

The current study was conducted in a periodontal referral-based practice and therefore represents a specific patient population, unrelated to the general dental medical population that does not receive periodontal referral. The patients are older than the general dental practice cohort, and this is a biased sample as these patients were referred to the periodontal practice for problems that were already screened by the referring dentist. Although these populations may not be representative of other clinical populations, the sample size was large to help identify the reliability of this novel questionnaire. The findings in the current patient population suggest that PROS may identify a majority of urgent dental care needs. Additional research is needed to determine this tool's clinical effectiveness in other clinical populations. The current findings from this study suggest that PROS may differentiate patients with more urgent dental needs in an alreadyreferred population.

\section{Conclusions}

An instrument that provides close correlation between patientreported oral health and intra-oral clinical exam could be useful in multiple scenarios where barriers to access to care exist. Potential applications include medically-complex patient populations such as cancer patients, in large-scale community based screenings and outreach to underserve and geographically remote populations. Additionally, non-dental health care professionals may find an instrument that accurately predicts oral health status useful to determine which patients may require urgent dental referral and intervention in high risk patient populations and when focused oral health training is lacking.

This study suggests that patients who have swelling in the mouth or jaw, third molar problems, jaw or tooth pain with biting, and loose teeth should be considered for referral for urgent care based on those symptoms alone. If the patient is medically complex and may receive medical treatment that may increase risk of infection from any source 
(including dental), or result in limitations of future options for care (specifically invasive and surgical procedures) complete oral and dental examination should be performed by experienced dental professionals. The PROS screening form presents a novel instrument that provides a reliable way to potentially identify people with urgent dental care needs based on patient reported dental and oral symptoms. The instrument is brief and simple to administer and can be used in a variety of settings when an oral exam by a trained dental professional is prohibited because of access to dental care or the urgency of medical care and should be evaluated in other clinical settings.

\section{Acknowledgement}

The authors would like to thank Salah Khudair for his assistance in data collection for this manuscript.

\section{Conflict of interest}

The Authors have no potential conflicts of interest to disclose.

\section{Appendix 1:}

\section{PRO Oral/Dental Screening Form for Oral Health}

Patient's name: Date:

Age:__ Gender: M/F PID:

Consult: Comprehensive / Specific

Tobacco use: NO YES week packs per day or

Alcohol use week

Oral status: NO _ drinks per day or

Tooth brushing. Twice a day

Tooth flossing/Interdental cleaning: __ Never___Irregular __ At least once a day

\section{Do you wear dentures?}

Do you have problems with your dentures? some teeth most teeth

Do you have problems with 3rd molars (wisdom teeth)?

Have you seen a dentist in the past year?

Have you had dental (tooth) pain in the past year?

Do you have any facial pain/ tooth pain now? YES

Do you have any mass or swelling in the mouth YES or jaw?

Do you have sores in your mouth? YES

Have you had any change in speech or in eating YES

due

to mouth problems?

Do you have difficulty swallowing solid food? YES

Do you have loose teeth?

Do you have bleeding in your mouth?

Do you have swollen gums?

Do you have any broken teeth or broken

fillings?

Do you have dry mouth?

Do you grind your teeth?
Do you have any jaw or tooth pain with biting? YES Is your jaw opening limited?

YES $\mathrm{NO}$ $\mathrm{NO}$

How would you describe your oral health? Poor

For office use only:

- $\quad$ Patient requires urgent care? YES

- If yes, cite reason:

o Pain requiring analgesics

o Swelling

o Suspicious lesion

Other:

\section{References}

1. Centers for Disease Control and Prevention DoOHU. (2009) Oral health: Preventing cavities, gum disease, and tooth loss.

2. Cohen LA, Bonito AJ, Akin DR, Manski RJ, Macek MD, et al. (2008) Toothache pain: a comparison of visits to physicians, emergency departments and dentists. $J$ Am Dent Assoc 139: 1205-1216. [Crossref]

3. Douglass AB, Douglass JM, Krol DM (2009) Educating pediatricians and family physicians in children's oral health. Acad Pediatr 9: 452-456. [Crossref]

4. Feinglass J, Nonzee NJ, Murphy KR, Endress R, Simon MA (2014) Access to care outcomes: A telephone interview study of a suburban safety net program for the uninsured. J Community Health 39: 108-117.

5. Neely M, Jones JA, Rich S, Gutierrez LS, Mehra P (2014) Effects of cuts in Medicaid on dental-related visits and costs at a safety-net hospital. Am J Public Health 104: e13-16. [Crossref]

6. Pajewski NM, Okunseri C (2014) Patterns of dental service utilization following nontraumatic dental condition visits to the emergency department in Wisconsin Medicaid. J Public Health Dent 74: 34-41. [Crossref]

7. Weinstein P (1998) Public health issues in early childhood caries. Community Dent Oral Epidemiol 26: 84-90. [Crossref]

8. Bergan EH, Tura BR, Lamas CC (2014) Impact of improvement in preoperative oral health on nosocomial pneumonia in a group of cardiac surgery patients: A single arm prospective intervention study. Intens Care Med 40: 23-31.

9. Lee HH, Lewis CW, Saltzman B, Starks H (2012) Visiting the emergency departmen for dental problems: trends in utilization, 2001 to 2008. Am J Public Health 102: e7783. [Crossref]

10. Committee on Oral Health Access to Services Board on Children Youth, and Families Board on Health Care Services. Institute of Medicine and National Research Council of the national academy. Improving access to oral health care for vulnerable and underserved populations. 2011: 229-254.

11. Cohen LA (2013) Expanding the physician's role in addressing the oral health of adults. Am J Public Health 103: 408-412. [Crossref]

12. Cohen LA, Bonito AJ, Eicheldinger C, Manski RJ, MacEk MD, Edwards RR, et al (2011) Comparison of patient visits to emergency departments, physician offices, and dental offices for dental problems and injuries. J Public Health Dent 71: 13-22.

13. Epstein JB, Parker IR, Epstein MS, Gupta A, Kutis S, et al. (2007) A survey of national cancer institute-designated comprehensive cancer centers' oral health supportive care practices and resources in the USA. Supportive Care Cancer 15: 357-62.

14. Epstein JB, Güneri P, Barasch A (2014) Appropriate and necessary oral care for people with cancer: guidance to obtain the right oral and dental care at the right time. Support Care Cancer 22: 1981-1988. [Crossref]

15. Guggenheimer J, Eghtesad B, Stock DJ (2003) Dental management of the (solid) organ transplant patient. Oral Surg Oral Med Oral Pathol Oral Radiol Endod 95: 383-389. [Crossref]

16. Jacques PF, Snow C, Dowdle M, Riley N, Mao K, et al. (2010) Oral health curricula 
in physician assistant programs: A survey of physician assistant program directors. $J$ Physic Assist Edu 21: 22-30.
17. http://www.cancer.gov/cancertopics/pdq/supportivecare/oralcomplications/ HealthProfessional/page3

Copyright: $\odot 2016$ Epstein JB. This is an open-access article distributed under the terms of the Creative Commons Attribution License, which permits unrestricted use, distribution, and reproduction in any medium, provided the original author and source are credited. 